Moroccan J. of Pure and Appl. Anal. (MJPAA)

Volume 5(2), 2019, Pages 263-278

ISSN: Online 2351-8227 - Print 2605-6364

DOI 10.2478/mjpaa-2019-0019

\title{
An Efficient Algorithm for Reconstruction Images Corrupted by Some Multiplicative Noises
}

\section{ZIAD $^{1}$, O. OUBBIH ${ }^{2}$ AND F. SNIBA ${ }^{3}$}

Aвstract. In this paper, we propose a novel hybrid model for restoration of images corrupted by multiplicative noise. Using a MAP estimator, we can derive a functional whose minimizer corresponds to the denoised image we want to recover. The energies studied here are inspired by image restoration with non linear variable exponent [1,2], and it is a combination of fast growth with respect to low gradient and slow growth when the gradient is large. We study a mathematical framework to prove the well posedness of the minimizer problem and we introduce the associated evolution problem, for which we derive numerical approaches. At last, compared experimental results distinctly demonstrate the superiority of the proposed model, in term of removing some muliplicative noise while preserving the edges and reducing the staircase effect.

Mathematics Subject Classification (2010). 68U10, 46E30, 65D18.

Key words and phrases. Multiplicative noise, Total variation, Image restoration, Nonlinear energy, Variable exponent, Numerical algorithm.

\section{Introduction}

Image denoising is a substantial image processing task, the purpose of an image denoising algorithm is to achieve both noise reduction and feature preservation. Traditionally, additive gaussian noise is the most used to corrupt images and several methods and techniques have

Received 05 October 2019 - Accepted: 24 December 2019.

(C) The Author(s) 2017. This article is published with open access by Sidi Mohamed Ben Abdallah University.

1,2,3 Ecole Supérieure de Technologie d'Essaouira, B.P. 383 Essaouira El Jadida, Essaouira.

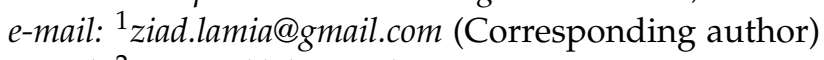

e-mail: ${ }^{2}$ omar.oubbih@usmba.ac.ma

e-mail: ${ }^{3}$ farahsniba@gmail.com . 
been used to reduce noise, see for instance $[1,2,4,16,18,19,20,21,24,28]$ and for other approaches in image denoising see for instance[10,12]. In this case, for a given original corrupted image $f$, the problem is then to recover $u$ from the data, which can be written as

$$
f=u \oplus \zeta
$$

where $\zeta$ represents the gaussian noise. However, in many real world applications, images are not often contaminated with additive gaussian noises. For example in digital images, specially when their acquisition system is based on photon detection, noise can be intensity dependent. This can be expressed with a multiplicative noise which appears in various image processing applications, such as in laser images, microscope images and medical ultrasonic images. Unlike the additive noise model, the presence of multiplicative noise destroys the information content in the original image to a great extent, and the image reconstruction problem under the multiplicative noise is evidently challenging. For a mathematical description of such problem, we assume that an image $u$ is a real function defined in a bounded open subset $\Omega$ of $\boldsymbol{R}^{2}$. The corrupted image $f$ is given by

$$
f=u \otimes \zeta
$$

where $\zeta$ represents the multiplicative noise. These category of noises are much more difficult to be removed from the degraded images, mainly not only because of their multiplicative nature, but also because of their distributions which are generally not Gaussian.

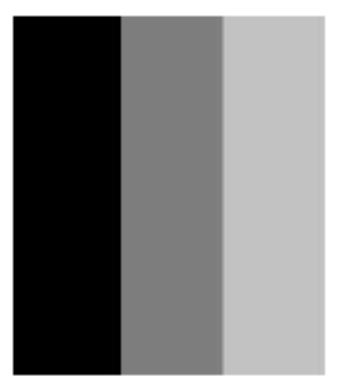

(a) Original image.

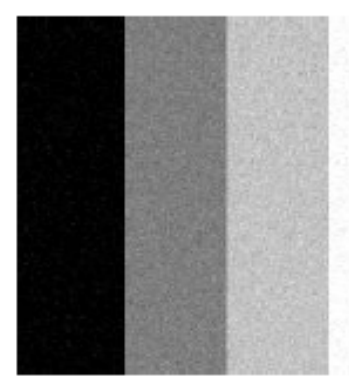

(b) Additive noise.

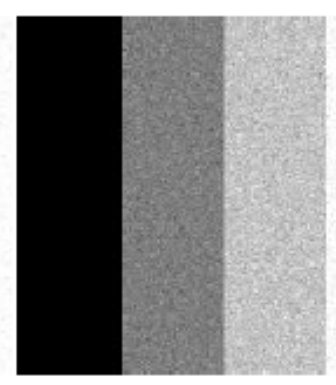

(c) Poisson noise.

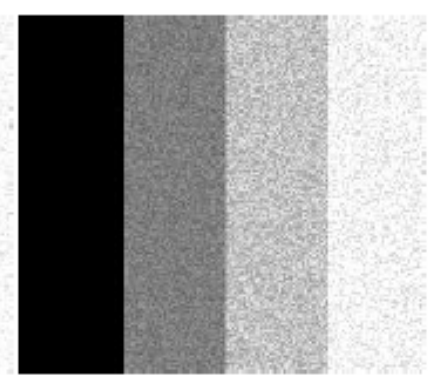

(d) Gamma noise.

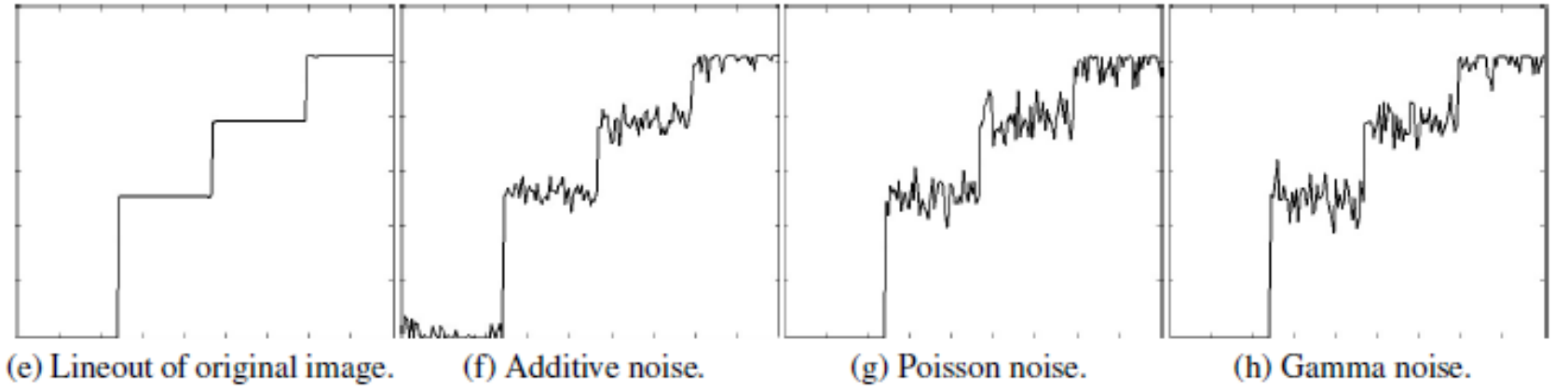

Figure 1: Comparison between corruption by additive and multiplicative noises.

The multiplicative noise has not yet been studied thoroughly. As far as we know, some approaches have been proposed to handle this multiplicative noise removal problems, such as wavelet approaches [11], filtering approaches [26, 27] and variational approaches [5, 17, 23, 24, 25]. Among the variational approaches, total variation (TV) based models seem to be rather 
appealing. It is well known that Total Variation (TV) can handle edges, but the images resulting from its application are usually piecewise constant, and have sometimes undesirable staircasing effect see for instance (cf. [2, 4, 6, 7, 13]), which causes images to look blocky. To overcome this, many regularization approaches (cf. [1, 2, 8, 9, 14, 15, 18, 19, 20, 24] and the references therein) has been suggested. The framework for 0rlicz energy has shown its effectiveness to overcoming this flaw [1,2]. In the light of these work and in order to study further the multiplicative (poisson or gamma) denoising models, we consider to minimize an appropriate functional with a variable exponent. Implemented by the gradient projection method involving Euler-Lagrange equations and artificial time evolution, one seek the solution of problem formally. Numerical experiments show that our model could be seen as this kind of application of variable exponent driven image processing to remove multiplicative noise and reduce the stair-casing effect.

The paper is organized in three consecutive parts. In Section II, we propose a nonlinear regularization of total variation model devoted to restore images contaminated with multiplicative (poisson or gamma) noises, we use a MAP estimator to derive a functional whose minimizer corresponds to the denoised image we want to restore, we prove the existence and the uniqueness of the minimizer we develop also an efficient algorithm to compute the numerical solution. Section III is dedicated to numerical simulation to prove the effectiveness of the proposed model and we display some comparison with other works. Finally, we conclude our paper in Section IV.

\section{The proposed Model}

Let $f$ be the intensity of the noisy image that was contaminated with multiplicative noise. The goal is to recover the true image $u$ defined on $\Omega$, an open bounded domain. Since the multiplicative noise can be signal dependent, and obeys a Poisson or Gamma distribution, we Recall that the distribution can be identified, with mean and standard deviation $\delta$, by :

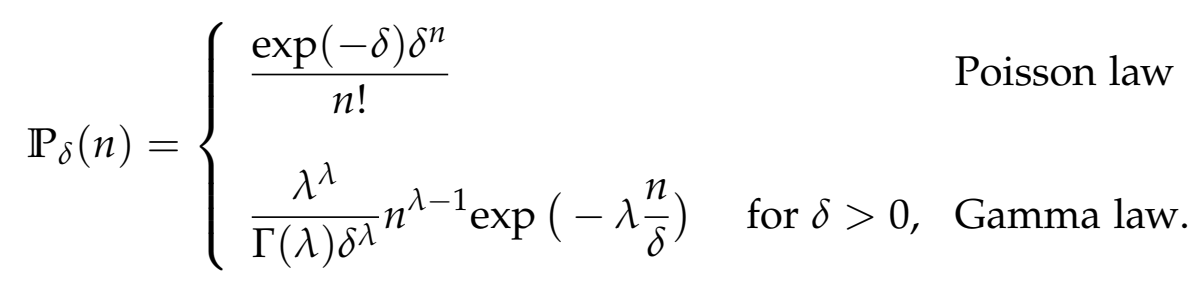

Assume that the noise in different pixels is statistically independent, and the joint probability of all the pixels is the product of the probabilities of the individual pixels, then

$$
\mathbb{P}(f \mid u)=\prod_{x \in \Omega} \mathbb{P}_{u(x)}(f(x))= \begin{cases}\prod_{x \in \Omega} \frac{u(x)^{f(x)} \exp (-u(x))}{f(x) !} & \text { Poisson } \\ \prod_{x \in \Omega} \frac{n^{n}}{\Gamma(n) u^{n}(x)} f(x)^{n-1} \exp \left(-n \frac{f(x)}{u(x)}\right) & \text { for } n>0, \text { Gamma. }\end{cases}
$$


For a given data $f$, the Bayes formula give

$$
\mathbb{P}(u \mid f)=\frac{\mathbb{P}(f \mid u) \mathbb{P}(u)}{\mathbb{P}(f)} .
$$

To determine an approximation to the unknown image $u$, we use the classical Maximum a Posteriori estimator (MAP) which maximizes the posterior density $\mathbb{P}(u \mid f)$, or minimizes the convex energy $-\log (\mathbb{P}(u \mid f))$, then thanks to (2.3) the minimizer $u^{*}$ satisfies

$$
u^{*} \in \arg \min _{u}\{-\log (\mathbb{P}(u \mid f))\}=\arg \min _{u}\{-\log (\mathbb{P}(f \mid u))-\log (\mathbb{P}(u)\} .
$$

To describe the prior Gibbs functions, we use the generalized formula, which is

$$
\mathbb{P}(u)=\exp \left(-\frac{1}{\lambda} \int_{\Omega} \Psi(x,|\nabla u|) d x\right),
$$

where $\lambda$ is a positive parameter and $\Psi$ is a non negative strictly convex function satisfying $\Psi(x, 0)=0$ a.e in $\Omega$ and

$$
\lim _{t \rightarrow+\infty} \Psi(x, t)=+\infty \quad \text { a.e in } \Omega .
$$

Thanks to (2.4), (2.2) and (2.5) the minimization problem can be rewritten as:

$$
\min _{u} \mathcal{F}(u):=\int_{\Omega} \mathcal{H}_{f}(u) d x+\frac{1}{\lambda} \int_{\Omega} \Psi(x,|\nabla u|) d x .
$$

A typical models are also Poisson or Gamma distributed data

$$
\mathcal{H}_{f}(u)= \begin{cases}u-f \log (u) & \text { Poisson noise } \\ \frac{f}{u}+\log (u) & \text { Gamma noise, }\end{cases}
$$

and the Euler-Lagrange equation associated to (2.6) is given by

$$
\operatorname{div}\left(\frac{\Phi(x,|\nabla u|) \nabla u}{|\nabla u|}\right)-\lambda \mathcal{M}_{f}(u)=0,
$$

where for a.e. $x \in \Omega, \Psi(x, s)=\int_{0}^{s} \Phi(x, t) d t$ and

$$
\mathcal{M}_{f}(u)= \begin{cases}\frac{f-u}{u} & \text { Poisson noise } \\ \frac{f-u}{u^{2}} & \text { Gamma noise. }\end{cases}
$$

Notice that a different choice of $\Phi$ correspond to different energy and models. In the context of additive Gaussian noise. Taking $\Phi(x, s)=\frac{s}{1+s^{2}}$ a.e. in $\Omega$, Perona and Malik have presented the first nonlinear partial differential equations model for denoising in a class of models commonly referred to as anisotropic diffusion. By choosing $\Phi(x, s)=1$ a.e. in $\Omega$, Rudin et al. ( $\mathrm{ROF}$ ) in [24] have proposed an alternative model called total variation model, that can protect the details of image better in the course of denoising. We recall that the Total variation model, 
is not well defined at locations where $|\nabla u|=0$ due to the presence of the term $\frac{1}{|\nabla u|}$ and it is known that the solution of this problem is defined in the non standard variational sense (cf. [3] ). The drawback of this model consists in creating stair-casing effect so that meanwhile various alternative regularizers were considered. In current work, we propose a new restoration model for multiplicative noise, by using a nonlinear regularization of total variation model, which is written as :

$$
\Phi(x, s)=\Phi_{\gamma}(x, s)=\left\{\begin{array}{ll}
\mu(x) s^{\alpha(x)-1} & \text { if } s \leq \varepsilon \\
\log ^{\gamma}(1+s) & \text { if } s>\varepsilon
\end{array} \quad \text { a.e. } x \in \Omega,\right.
$$

where $1<v \leq \alpha(x) \leq 2$. Here one can choose

$$
\alpha(x)=1+\frac{1}{1+\left|\nabla G_{\sigma} * f(x)\right|^{2}}, \mu(x)=\frac{\log ^{\gamma}(1+\varepsilon)}{\varepsilon^{\alpha(x)-1}}
$$

where $G_{\sigma}(x)=\frac{1}{\sigma} \exp \left(-|x|^{2} / 4 \sigma^{2}\right)$ is the Gaussian filter and $\sigma>0$ is a fixed parameter. The parameters $\varepsilon$ and $\gamma$ are nonnegatives. When $\gamma$ is close to 0 , we have

$$
\operatorname{div}\left(\Phi_{\gamma}(x,|\nabla u|) \frac{\nabla u}{|\nabla u|}\right) \rightarrow \operatorname{div}\left(\frac{\nabla u}{|\nabla u|}\right) \quad \text { in }[|\nabla u|>\varepsilon],
$$

and when $[|\nabla u| \leq \varepsilon]$, we observe that

$$
\operatorname{div}\left(\Phi_{\gamma}(x,|\nabla u|) \frac{\nabla u}{|\nabla u|}\right) \sim \operatorname{div}\left(|\nabla u|^{\alpha(x)-2} \nabla u\right) .
$$

That is, at edges where $|\nabla u|$ is large, the proposed operators can be seen as a regularization of TV operator. Our model is well posed in a weak sense and a numerical simulations substantially reduces the staircase effect.

Now, let $A(t)=t \log ^{\gamma}(1+t)$ and we recall that (cf. [2] for instance) $L_{A}(\Omega)$ is a Banach space under the norm

$$
\|u\|_{A, \Omega}=\inf \left\{\lambda>0: \int_{\Omega} A\left(\frac{u(x)}{\lambda}\right) d x \leq 1\right\} .
$$

The closure in $L_{A}(\Omega)$ of the set of bounded measurable functions with compact support in $\bar{\Omega}$ is denoted by $E_{A}(\Omega)$. The dual of $E_{A}(\Omega)$ can be identified with $L_{\bar{A}}(\Omega)$ by means of the pairing $\int_{\Omega} u(x) v(x) d x$, and the dual norm on $L_{\bar{A}}(\Omega)$ is equivalent to $\|\cdot\|_{\bar{A}, \Omega}$. The Orlicz-Sobolev space $W^{1} L_{A}(\Omega)$ is the space of all functions $u$ such that $u$ and its distributional derivatives up to order 1 lie in $L_{A}(\Omega)$. This is a Banach space under the norm

$$
\|u\|_{1, A, \Omega}=\sum_{|\alpha| \leq 1}\left\|D^{\alpha} u\right\|_{A, \Omega} .
$$

Thus $W^{1} L_{A}(\Omega)$ can be identified with subspaces of the product of $N+1$ copies of $L_{A}(\Omega)$. Denoting this product by $\Pi L_{A}$, we will use the weak topologies $\sigma\left(\Pi L_{A}, \Pi E_{\bar{A}}\right)$. 
Theorem 2.1. Let $f \in L^{\infty}(\Omega)$ with $\inf f>0$ be given. Then for every $\gamma \geq 0$, there exists a minimizer $u \in W^{1} L_{A}(\Omega)$ such that $0<\inf f \leq u \leq \sup f$ and

$$
\min _{v>0: v \in E} \mathcal{F}(v):=\int_{\Omega} \mathcal{H}_{f}(v) d x+\frac{1}{\lambda} \int_{\Omega} \Psi(x,|\nabla v|) d x
$$

where $E=W^{1} L_{A}(\Omega)$.

Proof: The functional $\mathcal{J}(v)=\mathcal{H}_{f}(v)$ takes its minimum at $v=f$ so that $\mathcal{F}(v)$ is bounded from below. Consequently, $\inf _{v>0: v \in E} \mathcal{F}(v)<\infty$. Now, we show that $\inf _{v>0: v \in E} \mathcal{F}(v)=\inf _{a \leq v \leq b: v \in E} \mathcal{F}(v)$, where $a=\inf f$ and $b=\operatorname{supf}$. It is not difficult to see that

$$
\Psi(x,|\nabla \min (v, \sup f)|) \leq \Psi(x,|\nabla v|) \text { and } \Psi(x,|\nabla \max (v, \inf f)|) \leq \Psi(x,|\nabla v|) .
$$

Using the monotonicity of $\mathcal{H}_{f}$ and following the same arguments as in Theorem 4.1 [5], we obtain

$$
\mathcal{H}_{f}(\min (v, \sup f)) \leq \mathcal{H}_{f}(v) \quad \text { and } \mathcal{H}_{f}(\max (v, \inf f)) \leq \mathcal{H}_{f}(v) .
$$

Consequently, we have

$$
\inf _{v>0: v \in E} \mathcal{F}(v)=\inf _{a \leq v \leq b: v \in E} \mathcal{F}(v)
$$

Now, taking $u_{n}$ a minimizing sequence of $\mathcal{F}$, then there exists an $M>0$ such that

$$
\int_{\Omega} \mathcal{H}_{f}\left(u_{n}\right)+\frac{1}{\lambda} \int_{\Omega} \Psi\left(x,\left|\nabla u_{n}\right|\right) d x \leq M \quad \text { for all } n \in N
$$

Since $\inf f \leq u_{n} \leq \sup f$ and $\mathcal{H}_{f} \in \mathcal{C}[\inf f, \sup f]$, we obtain that $\mathcal{H}_{f}\left(u_{n}\right)$ is bounded. Using (2.12), we get

$$
\frac{1}{\lambda} \int_{\Omega} \Psi\left(x,\left|\nabla u_{n}\right|\right) d x \leq M
$$

Then, we have

$$
\frac{1}{\alpha(x)} \int_{\left[\left|\nabla u_{n}\right| \leq \varepsilon\right]} \mu(x)\left|\nabla u_{n}\right|^{\alpha(x)} d x+\int_{\left[\left|\nabla u_{n}\right|>\varepsilon\right]} \log ^{\gamma}\left(1+\left|\nabla u_{n}\right|\right)\left|\nabla u_{n}\right| d x \leq \lambda M,
$$

Therefore, there exists a constant $C>0$ such that

$$
\int_{\Omega} A\left(\left|\nabla u_{n}\right|\right) d x=\int_{\Omega} \log ^{\gamma}\left(1+\left|\nabla u_{n}\right|\right)\left|\nabla u_{n}\right| d x \leq C .
$$

Therefore, since $\left(u_{n}\right)$ is bounded in $L_{A}(\Omega)$, we deduce that $\left(u_{n}\right)$ is bounded in $W^{1} L_{A}(\Omega)$ and there exist a subsequence of $u_{n}$ will be noted also by $u_{n}$ such that

$$
u_{n} \rightarrow u \text { weakly in } W^{1} L_{A}(\Omega) \text { for } \sigma\left(\prod L_{A}, \prod E_{\bar{A}}\right) .
$$

Using the convexity of $A$, we gets

$$
\mathcal{F}\left(\liminf _{n \rightarrow \infty} u_{n}\right) \leq \liminf _{n \rightarrow \infty} \mathcal{F}\left(u_{n}\right) .
$$

Hence, $u$ is a solution of problem (2.11) and the proof of Theorem is holds. 
Remark 2.1. In the case of poissonian noise i.e. $\mathcal{H}_{f}(u)=u-f \log (u)$. The uniqueness of the minimizer follows from the strict convexity of $\mathcal{H}_{f}$ for $f>0$, which gives the strict convexity of (2.11). Otherwise, for Gamma noise i.e. $\mathcal{H}_{f}(u)=\frac{f}{u}+\log (u)$, we have

$$
\mathcal{H}_{f}^{\prime \prime}(u)=\frac{2 f-u}{u^{3}} .
$$

We deduce that if $0<u<2 f$, then $\mathcal{H}_{f}$ is striclty convex which gives the uniqueness of a minimizer (2.11).

\section{Computational Approach}

In this section, we present the numerical approach and we analyze the performance of the proposed model applied to image denoising, in addition to a comparative study with some denoising techniques described in the literature. To solve numerically the problem (2.8), we uses a parabolic equation with time as an evolution parameter, or equivalently, the gradient descent method. In other word, we develop a numerical approximation to the following problem

$$
\begin{cases}\frac{\partial u}{\partial t}-\operatorname{div}\left(\frac{\Phi(x,|\nabla u|) \nabla u}{|\nabla u|}\right)+\lambda \mathcal{M}_{f}(u)=0 & \text { in } Q:=(0, T) \times \Omega \\ \frac{\Phi(x,|\nabla u|) \nabla u}{|\nabla u|} \cdot \vec{n}=0 & \text { in } \Sigma:=(0, T) \times \partial \Omega \\ u(0, x)=\frac{1}{|\Omega|} \int_{\Omega} f(x) d x & \text { in } \Omega,\end{cases}
$$

where $\vec{n}$ is the unit normal to the boundary. For simplicity, the problem (3.1) can be rewritten as follows:

$$
\frac{\partial u}{\partial t}-\operatorname{div}\left(\mu(x) \nabla u|\nabla u|^{\alpha(x)-2} \chi_{[|\nabla u| \leq \varepsilon]}+\frac{\nabla u}{|\nabla u|} \log ^{\gamma}(1+|\nabla u|) \chi_{[|\nabla u|>\varepsilon]}\right)+\lambda \mathcal{M}_{f}(u)=0 \quad \text { in } Q
$$

where $u$ satisfying the homogeneous boundary condition, $u(0)=\frac{1}{|\Omega|} \int_{\Omega} f(x) d x$ is the average of the degraded image and $u(., t)$ is the restored image with scale parameter $t$. Now, Setting

$$
p(t, x):=\mu(x) \nabla u|\nabla u|^{\alpha(x)-2} \chi_{[|\nabla u| \leq \varepsilon]}+\frac{\nabla u}{|\nabla u|} \log ^{\gamma}(1+|\nabla u|) \chi_{[|\nabla u|>\varepsilon]} .
$$

For computing numerically problem (3.1), we attempt to discretize it by finite difference method. Assuming $k$ to be the time step size and $h$ the spatial grid size, we discretize time and space as follows:

$$
\begin{gathered}
t_{n}=n k, n=0,1,2, \ldots \\
x_{i}=i h, i=0,1,2, \ldots, M, \\
y_{j}=j h, j=0,1,2, \ldots, N,
\end{gathered}
$$


where $M h \times N h$ is the size of the original image. Denote $u_{i, j}^{n}$ and $\lambda^{n}$ the approximations of $u\left(t_{n}, x_{i}, y_{j}\right)$ and $/ \lambda\left(t_{n}\right)$ respectively. We define the discrete approximation:

$$
\begin{aligned}
& \nabla_{x}^{+} u_{i, j}^{n}=\frac{u_{i+1, j}^{n}-u_{i, j}^{n}}{h}, \nabla_{x}^{-} u_{i, j}^{n}=\frac{u_{i, j}^{n}-u_{i-1, j}^{n}}{h}, \\
& \nabla_{y}^{+} u_{i, j}^{n}=\frac{u_{i, j+1}^{n}-u_{i, j}^{n}}{h}, \nabla_{y}^{-} u_{i, j}^{n}=\frac{u_{i, j}^{n}-u_{i, j-1}^{n}}{h} .
\end{aligned}
$$

Using the following notation for simplicity:

$$
\left|\nabla u_{i, j}^{n}\right|=\sqrt{\left(\nabla_{x}^{+} u_{i, j}^{n}\right)^{2}+\left(\nabla_{y}^{+} u_{i, j}^{n}\right)^{2}} .
$$

Denote $\alpha_{i, j}$ and $\mu_{i, j}$ the approximations of $\alpha\left(x_{i}, y_{j}\right)$ and $\mu\left(x_{i}, y_{j}\right)$ respectively:

$$
\alpha_{i, j}=1+\frac{1}{1+\left|\nabla G_{\sigma} * f_{i, j}\right|^{2}}, \mu_{i, j}=\frac{\log ^{\gamma}(1+\varepsilon)}{\varepsilon^{\alpha_{i, j}-1}} .
$$

We denote by $\operatorname{div}(p)_{i, j}^{n}$ the approximations of $\operatorname{div}\left(p\left(t_{n}, x_{i}, y_{j}\right)\right)$ defined by:

$$
\begin{aligned}
\operatorname{div}(p)_{i, j}^{n} & =\nabla_{x}^{-}\left(\mu_{i, j} \nabla_{x}^{+} u_{i, j}^{n}\left|\nabla u_{i, j}^{n}\right|^{\alpha(x)-2} \chi_{\left[\left|\nabla u_{i, j}^{n}\right| \leq \varepsilon\right]}+\frac{\nabla_{x}^{+} u_{i, j}^{n}}{\left|\nabla u_{i, j}^{n}\right|} \log ^{\gamma}\left(1+\left|\nabla u_{i, j}^{n}\right|\right) \chi_{\left[\left|\nabla u_{i, j}^{n}\right|>\varepsilon\right]}\right) \\
& +\nabla_{y}^{-}\left(\mu_{i, j} \nabla_{y}^{+} u_{i, j}^{n}\left|\nabla u_{i, j}^{n}\right|^{\alpha(x)-2} \chi_{\left[\left|\nabla u_{i, j}^{n}\right| \leq \varepsilon\right]}+\frac{\nabla_{y}^{+} u_{i, j}^{n}}{\left|\nabla u_{i, j}^{n}\right|} \log ^{\gamma}\left(1+\left|\nabla u_{i, j}^{n}\right|\right) \chi_{\left[\left|\nabla u_{i, j}^{n}\right|>\varepsilon\right]}\right) .
\end{aligned}
$$

In the sequel, we present numerical results obtained by applying our proposed algorithm to image denoising. The first experiments are done to assess the performance of the proposed algorithm in order to remove noise. Afterwards, the performance of the proposed method is compared with that of TV that was adapted to multiplicative noise.

3.1. Gamma denoising. In this part we are dealing with the type of noise that follows a gamma distribution. For simplicity, the problem (3.1) can be written as follows:

$$
\begin{cases}\frac{\partial u}{\partial t}-\operatorname{div}(p(x, t))+\lambda\left(\frac{f-u}{u^{2}}\right)=0 & \text { in } Q \\ p(x, t)=\mu(x) \nabla u|\nabla u|^{\alpha(x)-2} \chi_{[|\nabla u| \leq \varepsilon]}+\frac{\nabla u}{|\nabla u|} \log ^{\gamma}(1+|\nabla u|) \chi_{[|\nabla u|>\varepsilon]} & \text { in } Q \\ u(0)=\frac{1}{|\Omega|} \int_{\Omega} f(x) d x & \text { in } \Omega\end{cases}
$$

The algorithm of the problem (3.2) could be written as: 


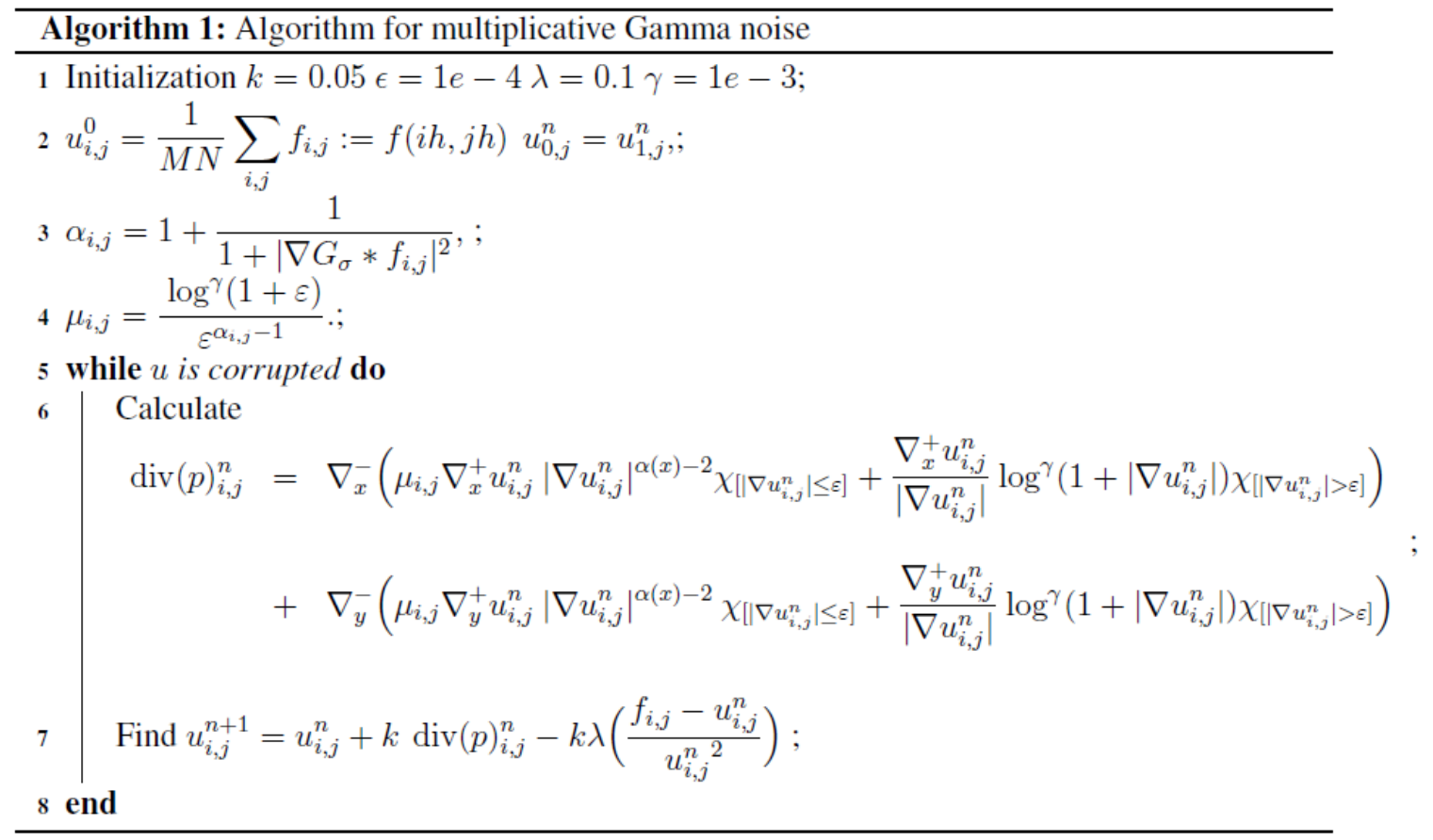

Firstly an efficiency test is provided to prove the effectiveness of our algorithm in denoising images contaminated by speckle noise, after that a comparison with the TV Model is done. Figure 2 presents the results of denoising images contaminated by speckle noise we set $\lambda=0.1$, $\varepsilon=1 e-4, k=0.05, \gamma=1 e-3$ and the number of iterations $d=300$.
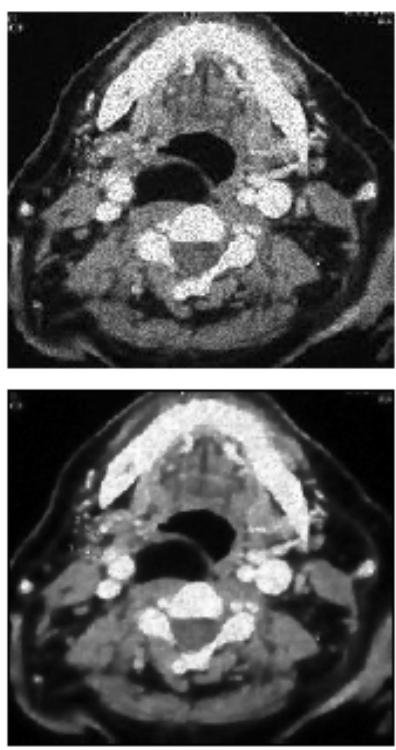

(a)
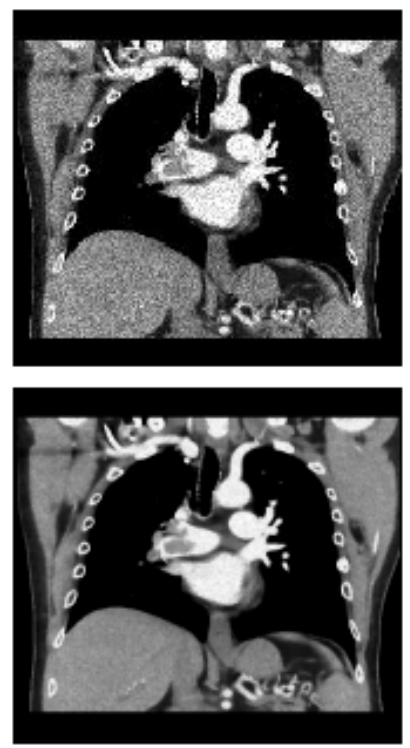

(b)
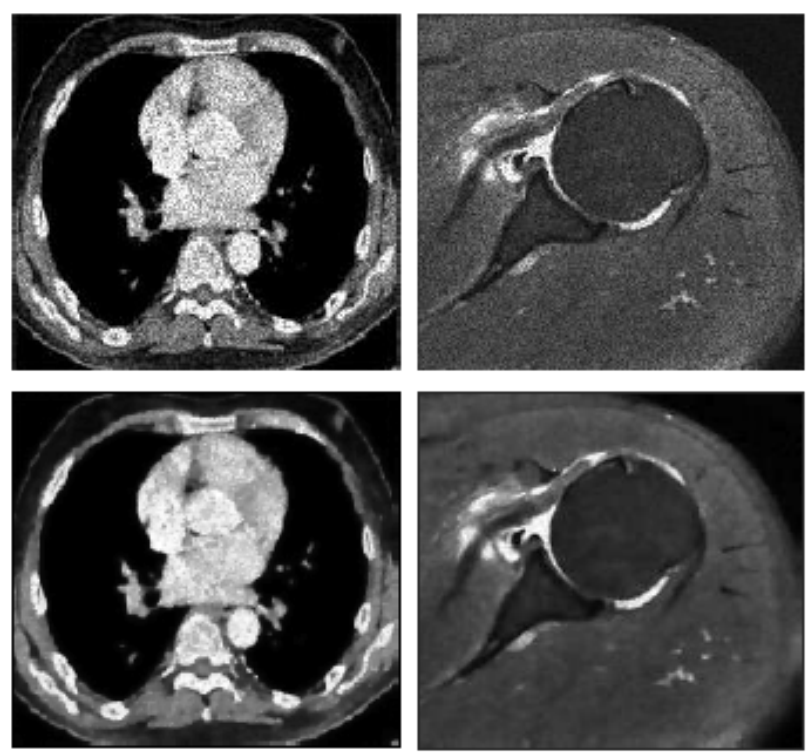

(c)

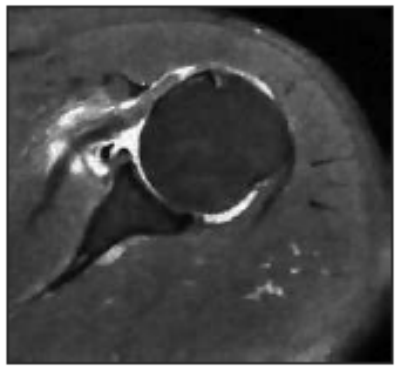

(d)

Figure 2: First row : Noisy images(Speckle noise) and second row : Restored ones. 
The previous experiments (Fig : 2) proved that the algorithm denoised the medical images corrupted by Speckle noise. To quantify the restoration quality of the results of the previous experiment, the performance parameters PSNR and SNR values are provided (Table 1) wich are defined as follows:

$$
\mathrm{PSNR}=10 \log _{10}\left(\frac{255^{2} M N}{\left\|u_{0}-u\right\|_{2}^{2}}\right) d B, \quad \mathrm{SNR}=\log _{10}\left(\frac{\sigma_{u}}{\sigma_{n}}\right) d B,
$$

where $u_{0}, u$ and $M \times N$ are the original image, the restored image and the size of the image, respectively. $\sigma_{u}$ and $\sigma_{n}$ are the signal and noise standard deviations, respectively.

\begin{tabular}{|l|c|c||c|c|c|c||c|c|}
\hline & \multicolumn{2}{|c|}{$(\mathrm{a})$} & \multicolumn{2}{c|}{ (b) } & \multicolumn{2}{c|}{ (c) } & \multicolumn{2}{c|}{$(\mathrm{d})$} \\
\cline { 2 - 9 } & PSNR & SNR & PSNR & SNR & PSNR & SNR & PSNR & SNR \\
\hline Noisy & 23.7102 & 12.5871 & 23.8183 & 13.5204 & 23.5966 & 13.4867 & 25.3364 & 7.2245 \\
Restored & 27.7291 & 16.6275 & 29.3936 & 19.1498 & 25.8430 & 15.7478 & 30.5838 & 12.4715 \\
\hline
\end{tabular}

TABLE 1. PSNR and SNR values of denoising results of Figure ??

In figure 3, we provide the results of denoising colored images contaminated by Speckle noise that follows a Gamma distribution.
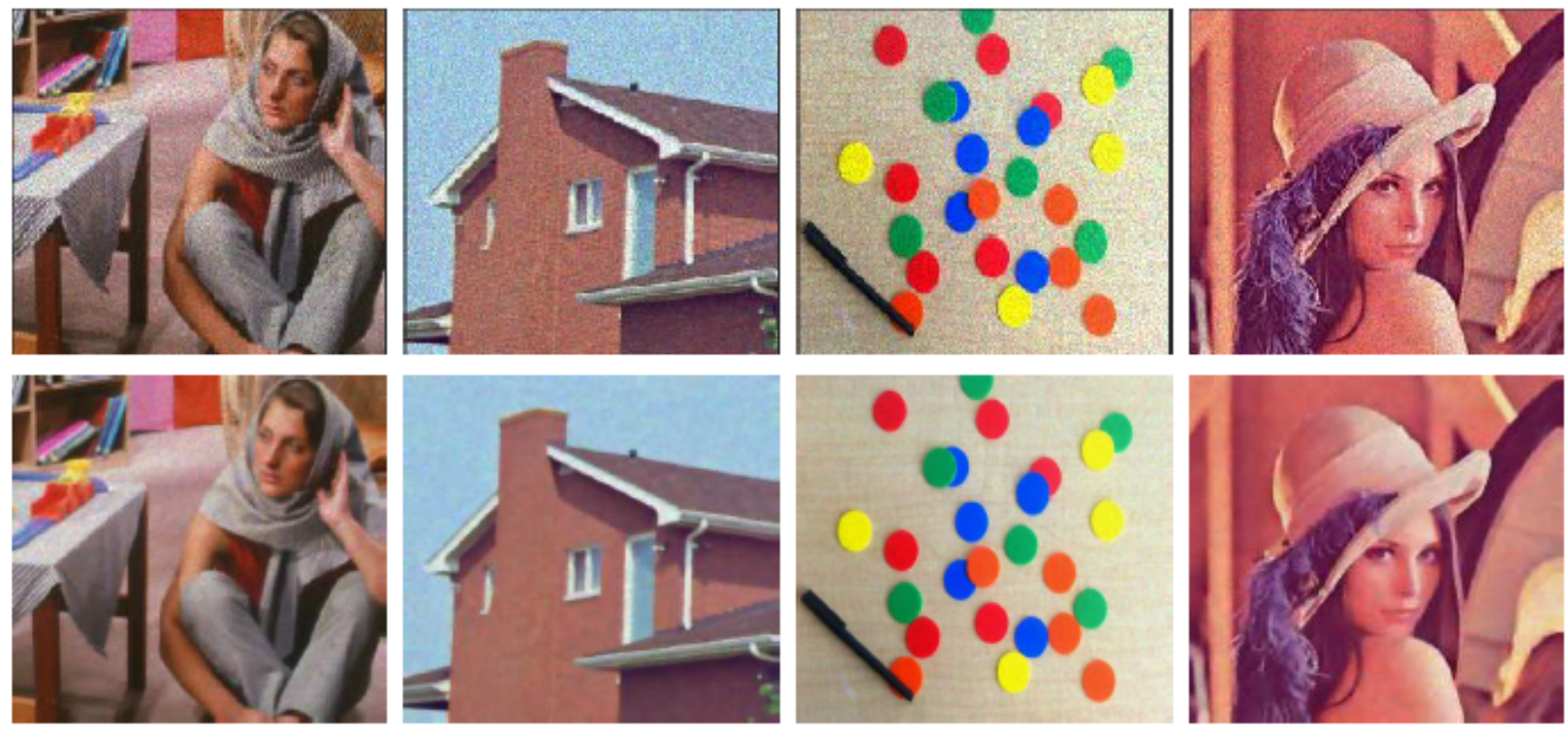

Figure 3: First row : Noisy colored images (Speckle noise) and second row : Restored ones.

After that, we will compare our algorithm with the ROF Model but with a fidelity term suitable for images corrupted by Gamma noise (Fig: 4). We take $\lambda=0.01$, same time stamp $k=0.05$ and the number of iterations $d=150$. 

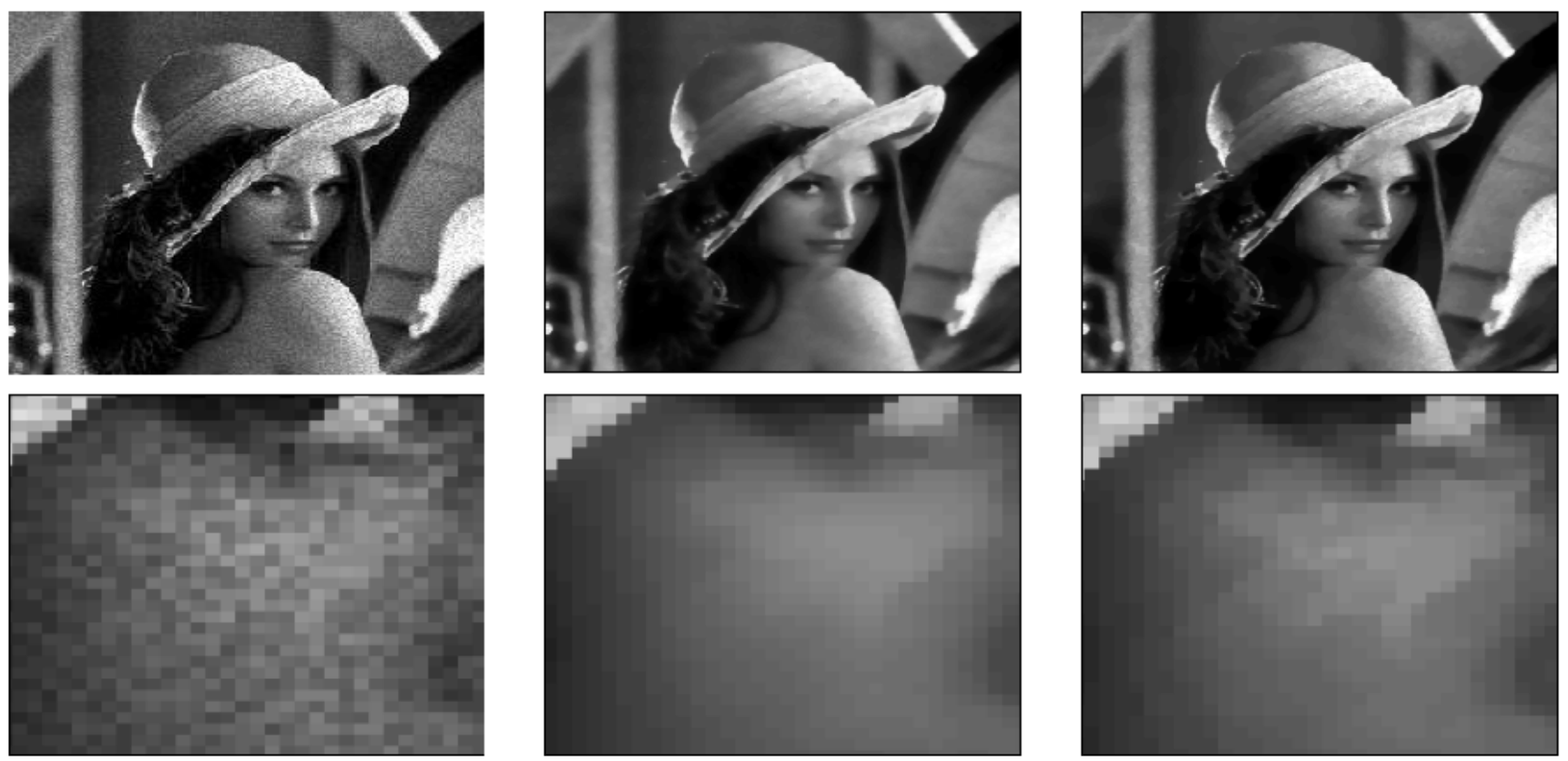

Figure 4: Left to right : Noisy image (Speckle noise), image restored by our algorithm, image restored by TV. Second row: zoom of the first row images

Figure 4, which displays the zoom (the check of lena), accessed that the proposed model avoids the Stair-Casing phenomena, whereas in the image resulting from the application of TV, StairCasing is present.

3.2. Poisson denoising model. Finding a suitable value for the fidelity parameter $\lambda$ is quit difficult, thus we will provide an automatic procedure to calculate $\lambda$. For this, using the approach comes from the fact that it is merely the gradient-projection method of Rosen [22]. Assume that the standard deviation of the noise $\eta$ is $\sigma$, we have

$$
\int_{\Omega}\left(\frac{f}{u}-1\right) d x=0 \text { and } \int_{\Omega}\left(\frac{f}{u}-1\right)^{2} d x=|\Omega| \sigma^{2} .
$$

Formally, multiplying the equation (2.8) by $\left(\frac{f-u}{u}\right)$ and integrating over $\Omega$, we obtain

$$
\lambda(t)=\frac{\int_{\Omega} \operatorname{div}\left(\frac{\Phi(x,|\nabla u|) \nabla u}{|\nabla u|}\right)\left(\frac{f-u}{u}\right)}{\int_{\Omega}\left(\frac{f-u}{u}\right)^{2} d x} .
$$

Thanks to (3.4), we see that $\int_{\Omega}\left(\frac{f-u}{u}\right)^{2} d x=|\Omega| \sigma^{2}$ in both cases, and the equations (3.1) and (3.5) give a dynamic of the value of $\lambda(t)$. For simplicity, considering Poisson noise, the 
problem (3.1) can be rewritten as follows:

$$
\begin{cases}\frac{\partial u}{\partial t}-\operatorname{div}(p(x, t))+\lambda(t)\left(\frac{f-u}{u}\right)=0 & \text { in } Q \\ p(x, t)=\mu(x) \nabla u|\nabla u|^{\alpha(x)-2} \chi_{[|\nabla u| \leq \varepsilon]}+\frac{\nabla u}{|\nabla u|} \log ^{\gamma}(1+|\nabla u|) \chi_{[|\nabla u|>\varepsilon]} & \text { in } Q \\ \lambda(t)=\frac{1}{|\Omega| \sigma^{2}} \int_{\Omega} \operatorname{div}(p(x, t))\left(\frac{f-u}{u}\right) d x & \text { in }(0, T) \\ u(0)=\frac{1}{|\Omega|} \int_{\Omega} f(x) d x & \text { in } \Omega\end{cases}
$$

The algorithm of the problem (3.6) could be written as:

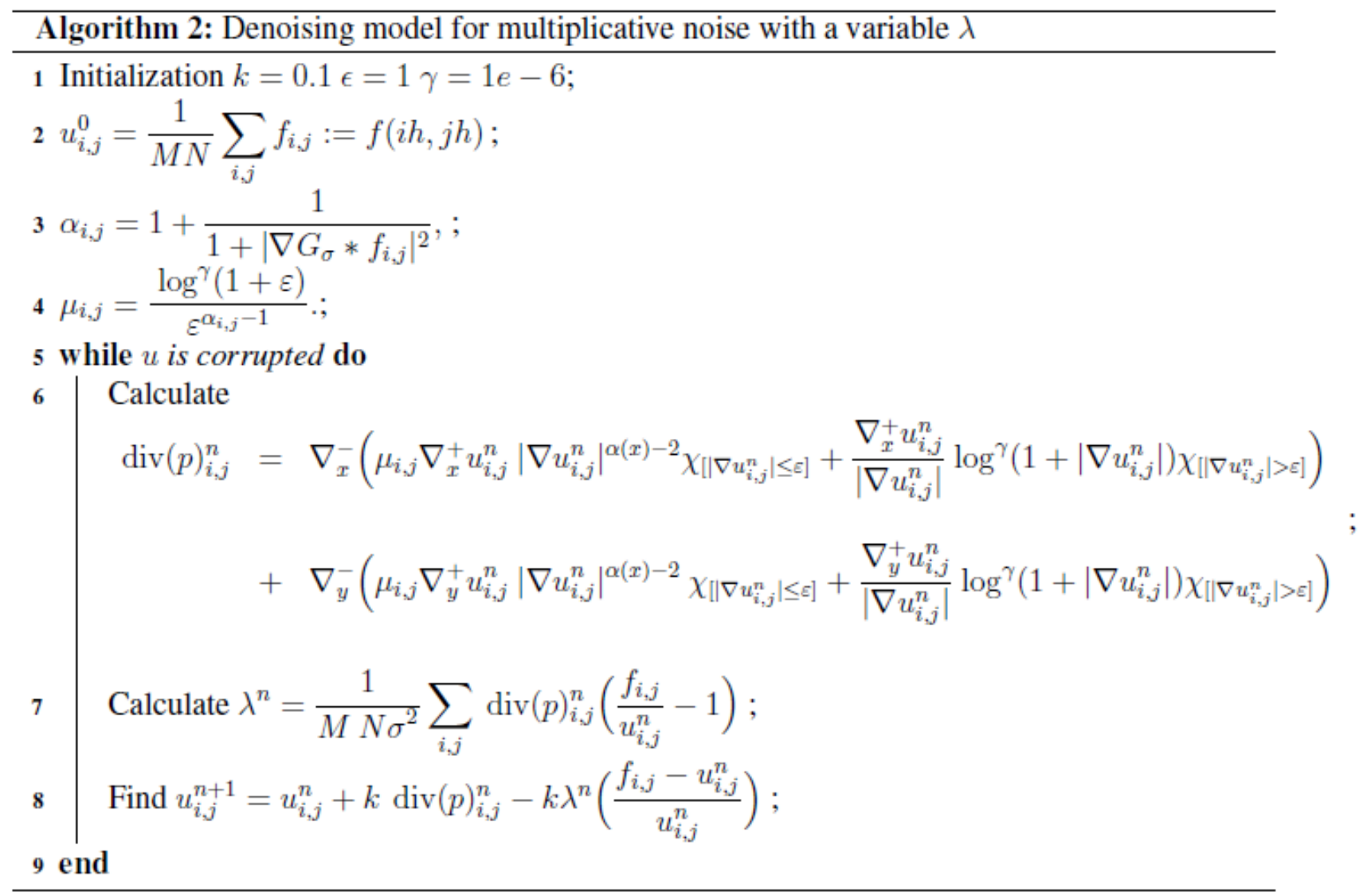

We test the proposed method on four different images, corrupted by a multiplicative noise, Figure 5 shows the results of denoising images contaminated by poisson noise. The corrupted images by Poisson noise are first presented and then the ones restored with our algorithm. 

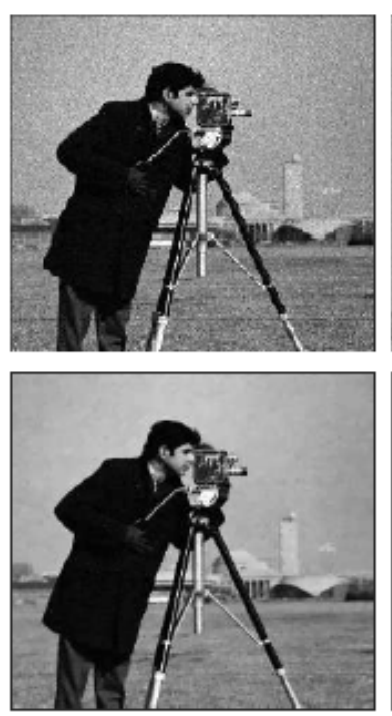

(a)
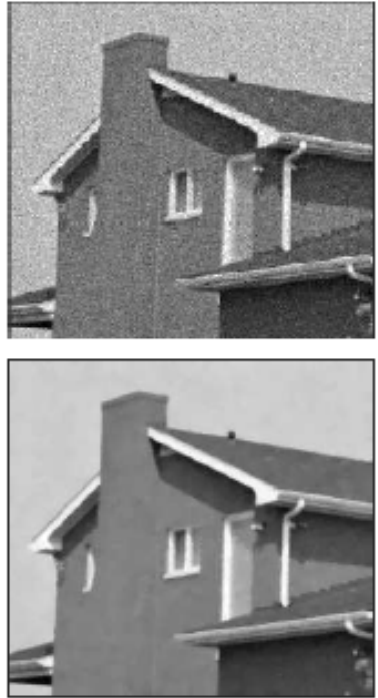

(b)
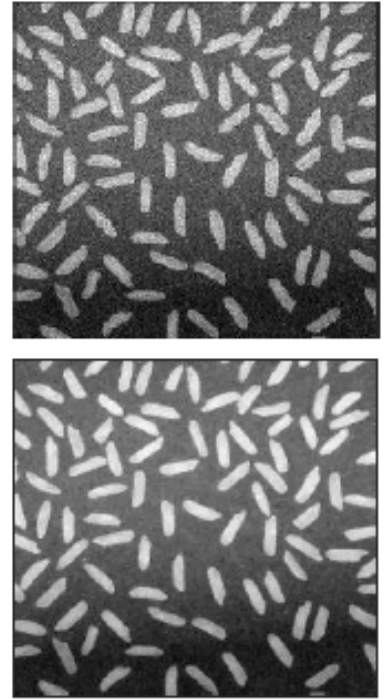

(c)
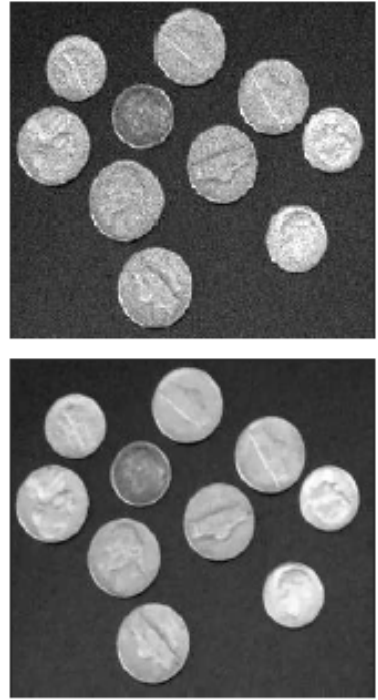

(d)

Figure 5: First row : Noisy images (Speckle noise) and second row: Restored ones.

The previous experiments proved that the algorithm is efficient against Poisson noise. After that, the PSNR and SNR values of the previous experiment (fig 5) are presented in Table 2.

\begin{tabular}{|l|c|c|c|c|c|c||c|c||}
\hline \multirow{2}{*}{} & \multicolumn{2}{|c|}{$(\mathrm{a})$} & \multicolumn{2}{c|}{$(\mathrm{b})$} & \multicolumn{2}{c|}{ (c) } & \multicolumn{2}{c|}{$(\mathrm{d})$} \\
\cline { 2 - 9 } & PSNR & SNR & PSNR & SNR & PSNR & SNR & PSNR & SNR \\
\hline Noisy & 27.3786 & 15.1433 & 26.7815 & 11.9099 & 27.6835 & 12.1192 & 28.0392 & 14.8547 \\
Restored & 32.2912 & 20.0908 & 33.3475 & 18.4970 & 31.7809 & 16.2443 & 32.9321 & 19.7734 \\
\hline
\end{tabular}

TABLE 2. PSNR and SNR values of figures 5

The SNR and PSNR values accessed the effectiveness of our algorithm in term of denoising images contaminated by Poisson noise. In the sequel, we will compare our algorithm with the TV model, adapted to denoise images corrupted by Poisson noise. We take $\lambda=0.01$ for TV, $\epsilon=25, \gamma=1 e-3$, same time stamp $d t=0.05$ and the number of iterations $d=20$.
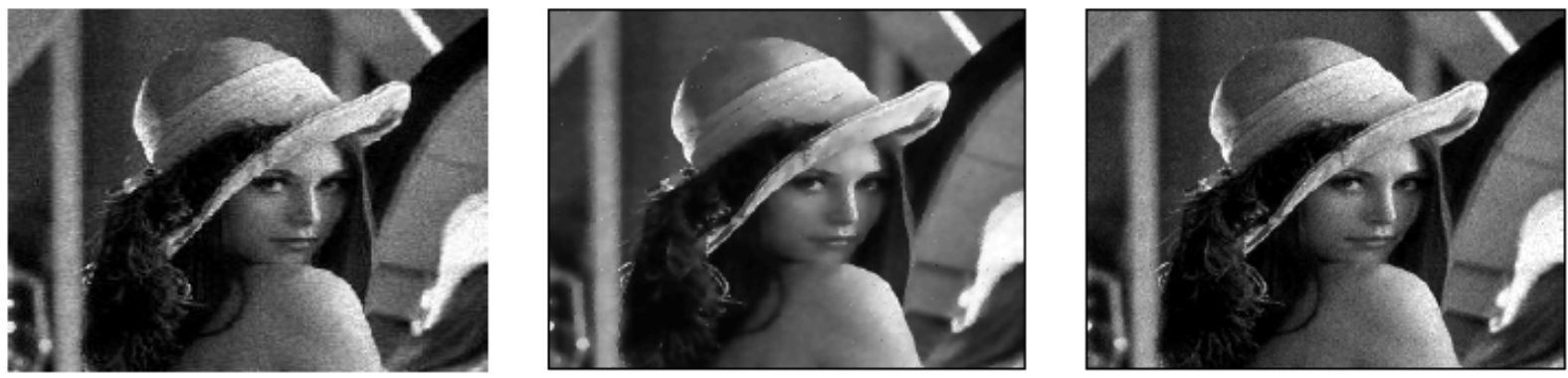

Figure 6: Left to right : Noisy images (Poisson noise), image restored by our algorithm and image restored by TV. 
It is obvious that the image resulting from the application of $\mathrm{Tv}$ is still noisy that's why we keep same parameters for our algorithm and increase the number of iterations of the TV until getting better results.
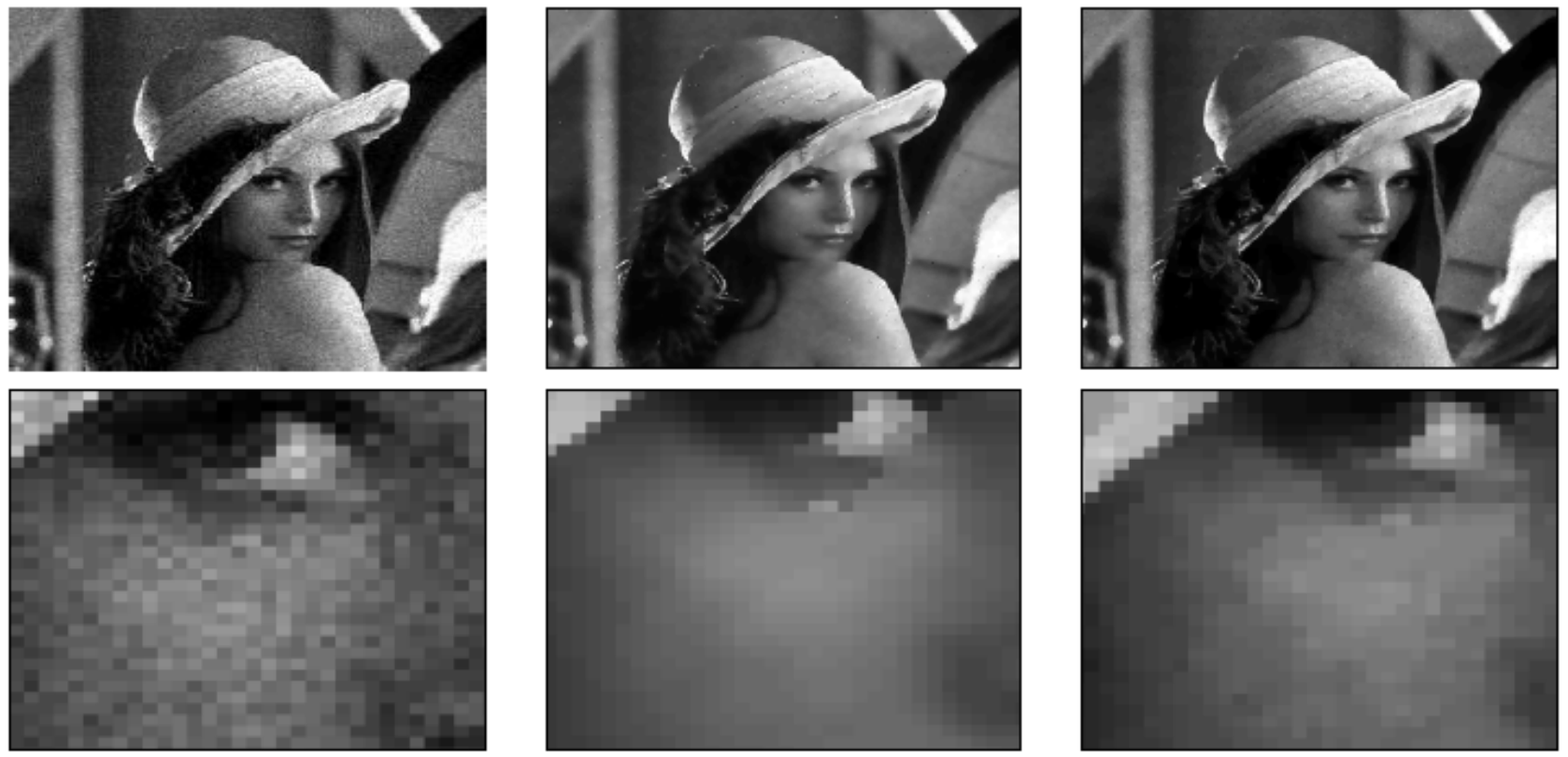

Figure 7: First row: Noisy images (Poisson noise), image restored by our algorithm, image restored by TV.

Second row : zoom of noisy image, zoom of image restored by our algorithm, zoom of image restored by TV.

The zoom (Fig : 7) shows that the main drawback of TV is avoided by our model. Besides, the image resulting from the application of our algorithm is denoised in a small number of iterations.

\section{Conclusion}

In this paper, we have proposed an hybrid model for restoration of images corrupted by multiplicative noise. Specially Gamma or Poisson distributed noises, the use of our model profits from the benefits of variable exponent to help removing some multiplicative noise while preserving the edges, mainly reducing the staircase effect and helping the solution to a quick convergence.

\section{References}

[1] R. Aboulaich, D. Meskine and A. Souissi, New diffusion models in image processing, Comput. Math. Appl. 56, (2008), 874-882.

[2] L. Afraites, A. Atlas, F. Karami and D. Meskine, Some class of parabolic systems applied to image processing,

Discrete \& Continuous Dynamical Systems - Series B . 21, (2016), 1671-1687. 
[3] F. Andreu, C. Ballester, V. Caselles and J.L. Mazòn, Minimizing total variation flow, Differential Integral Equations, 14 (2001), no. 3, 321-360.

[4] A. Atlas , F. Karami and D. Meskine, The peronamalik inequality and application to image denoising, Nonlinear Analysis: Real World Applications, 18 (2014), pp. 57-68.

[5] G. Aubert and J.F. Aujol, A variational approach to removing multiplicative noise, SIAM J. Appl. Math., 68 (2008), pp. 925-946.

[6] T. F. Chan, S. Esedoglu and F. E. Park, Image decomposition combining staircase reduction and texture extraction, Journal of Visual Communication and Image Representation, 18 (2007), 464-486.

[7] T. F. Chan, S. Esedoglu and F. E. Park, A fourth order dual method for staircase reduction in texture extraction and image restoration problems, 2010 IEEE International Conference on Image Processing, (2010), 4137-4140.

[8] S.G. Chang, B. Yu, M. Vetterli, Adaptive Wavelet Thresholding for Image Denoising and Compression, IEEE Trans. Image Process, Vol.9 (2000)(9), No.9, 1532-1546.

[9] Y. Chen, S. Levine, M. Rao, Variable exponent, linear growth functionals in image restoration, SIAM J. Appl. Math, 66 (2006), no. 4, 1383-1406.

[10] P. Dambra and G. Tartaglione, Solution of ambrosiotortorelli model for image segmentation by generalized relaxation method, Communications in Nonlinear Science and Numerical Simulation, 20 (2015), pp. 819-831.

[11] D. Donoho, Nonlinear wavelet methods for recovery of signals, densities and spectra from indirect and noisy data, Proceedings of Symposia in Applied Mathematics:Different perspectives on wavelets, Amer. Math. Soc. (1993), 173-205.

[12] A. Elmoataz, X. Desquesnes, and O. Lezoray, Non-local morphological pdes and p-laplacian equation on graphs with applications in image processing and machine learning, J. Sel. Topics Signal Processing, 6 (2012), pp. 764-779.

[13] P. Guidotti, J. Lambers, Two new nonlinear nonlocal diffusions for noise reduction, J. Math. Imaging Vision 33 (1) (2009) 25-37.

[14] P. Harjulehto, P.A. Hasto, V. Latvala, Minimizers of the variable exponent, non-uniformly convex Dirichlet energy, J. Math. Pures Appl. (9):89, (2008), 174-197.

[15] F. Karami, K. Sadik, and L. Ziad, A variable exponent nonlocal $p(x)$-laplacian equation for image restoration, Computers and Mathematics with Applications, 75 (2018), pp. 534546.

[16] F. Karami, L. Ziad, and K. Sadik, A splitting algorithm for a novel regularization of perona-malik and application to image restoration, EURASIP Journal on Advances in Signal Processing, 2017 (2017).

[17] R. Chartrand and T.J. Asaki, A variational approach to reconstructing images corrupted by Poisson noise, J. Math. Imaging Vision. 27, (2007), 257-263.

[18] X. Liu, L. Huang, A new nonlocal total variation regularization algorithm for image denoising, Mathematics and Computers in Simulation, (2014), 97, 224-233.

[19] A. Maleki, M. Narayan, AND R. G. Baraniuk, Anisotropic nonlocal means denoising, Applied and Computational Harmonic Analysis, 35 (2013), pp. 452-482.

[20] S. Oh, H. Woo, S. Yun, and M. Kang, Non-convex hybrid total variation for image denoising, J. Vis. Comun. Image Represent., 24 (2013), pp. 332-344.

[21] P. Perona, J. Malik, Scale-space and edge detection using anisotropic diffusion, IEEE Trans. Pattern Anal. Mach. Intell, (1990), 12(7), 629-639.

[22] J.G. Rosen, The gradient Projection Method for Nonlinear Programming. Part II. Nonlinear Constraints, Journal of the Society for Industrial and Applied Mathematics, 9 (1961), 514-532.

[23] L. Rudin, P.L. Lions, S. Osher, Multiplicative denoising and deblurring: theory and algorithms, in: S. Osher, N. Paragios (Eds.), Geometric Level Set Methods in Imaging, Vision, and Graphics, Springer, New York, 2003, pp. 103-119.

[24] L. Rudin, S. Osher, E. Fatemi, Nonlinear total variation based noise removal algorithms, Physica D 60, (1992), 259-268.

[25] J. Shi, S. Osher, A nonlinear inverse scale space method for a convex multiplicative noise models, SIAM J. Imaging Sci. 1 (2008) 294-321.

[26] K. Timmermann, R. Novak, Multiscale modeling and estimation of Poisson processes with applications to photonlimited imaging, IEEE Trans. Inf. Theory. 45, (1999), 846-862. 
[27] A. Trubuil and C. Kervrann, An adaptive window approach for poisson noise reduction and structure preserving in confocal microscopy, in 2004 2nd IEEE International Symposium on Biomedical Imaging: Nano to Macro (IEEE Cat No. 04EX821), Vol. 1, 2004, pp. 788-791.

[28] Y. Wang, W. Ren, H. Wang, Anisotropic second and fourth order diffusion models based on Convolutional Virtual Electric Field for image denoising, Computers and Mathematics with Applications, (2013), 66, 1729-1742. 